BMJ Paediatrics Open

\title{
Hybrid type 1 effectiveness/ implementation trial of the international Guide for Monitoring Child Development: protocol for a cluster-randomised controlled trial
}

\author{
Abhishek Raut (D) , ${ }^{1}$ Revan Mustafayev (D) , ${ }^{2}$ Roopa Srinivasan (D) , ${ }^{3}$ Anita Chary, ${ }^{4}$ \\ Ilgi Ertem (D) , ${ }^{5}$ Maria del Pilar Grazioso, ${ }^{6}$ Subodh Gupta (D) , \\ Vibha Krishnamurthy, ${ }^{3}$ Chunling Lu (D) , 7,8 Chetna Maliye, ${ }^{1}$ Ann C Miller (D) , 9 \\ Bradley H Wagenaar, ${ }^{10,11}$ Peter Rohloff (i) ${ }^{4,7}$
}

To cite: Raut A, Mustafayev R, Srinivasan $R$, et al. Hybrid type 1 effectiveness/implementation trial of the international Guide for Monitoring Child Development: protocol for a cluster-randomised controlled trial. BMJ Paediatrics Open 2021;5:e001254. doi:10.1136/ bmjpo-2021-001254

- Additional supplemental material is published online only. To view, please visit the journal online (http://dx.doi.org/ 10.1136/bmjpo-2021-001254).

Received 9 August 2021 Accepted 13 August 2021

\section{Check for updates}

(c) Author(s) (or their employer(s)) 2021. Re-use permitted under CC BY-NC. No commercial re-use. See rights and permissions. Published by BMJ.

For numbered affiliations see end of article.

Correspondence to Dr Peter Rohloff; prohloff@bwh. harvard.edu

\section{ABSTRACT}

Introduction More than $40 \%$ of children under 5 years of age in low-income and middle-income countries are at risk of not reaching their developmental potential. The international Guide for Monitoring Child Development (GMCD) early intervention package is a comprehensive programme to address developmental difficulties using an individualised intervention plan for young children and their families. We will conduct a hybrid type 1 effectiveness-implementation evaluation of the GMCD intervention in rural India and Guatemala.

Methods and analysis Using a cluster-randomised design, 624 children aged 0-24 months in 52 clusters (26 in India, 26 in Guatemala) will be assigned to usual care or the GMCD intervention plus usual care delivered by frontline workers for 12 months. After 12 months, the usual care arm will cross over to the intervention, which will continue for 12 additional months (24 total). The intervention will be delivered using a digital mobile device interface. Effectiveness will be assessed for developmental functioning (Bayley Scales of Infant Development, 3rd edition) and nurturing care (Home Observation for Measurement of the Environment Scale) outcomes. Implementation will be assessed using the Reach, Effectiveness, Adoption, Implementation, Maintenance framework. Explanatory qualitative analysis guided by the Consolidated Framework for Implementation Research will explore determinants between clusters with high versus low implementation effectiveness. Ethics and dissemination The study has been approved by the Institutional Review Boards of Brigham and Women's Hospital, Mahatma Gandhi Institute of Medical Sciences and Maya Health Alliance; and by the Indian Council of Medical Research/Health Ministry Screening Committee. Key study findings will be published in international open-access journals.

Trial registration number NCT04665297, CTRI/2020/12/029748.

Protocol version 1.0 (12 November 2020).

\section{INTRODUCTION}

In low/middle-income countries (LMICs), over $40 \%$ of children under age 5 are at risk

\section{What is already known on this topic?}

More than $40 \%$ of children under 5 years of age are at risk of not achieving their developmental potential.

- Caregiver support interventions are an evidencebased approach to support early child development.

- The Guide for Monitoring Child Development (GMCD) early intervention package is designed for use in low-income and middle-income countries, but realworld effectiveness data are lacking.

\section{What this study hopes to add?}

Effectiveness data for the GMCD from rural India and Guatemala for child development and nurturing care outcomes.

- The study includes detailed comparative implementation assessments which will identify important barriers and facilitators to the use of GMCD.

of not reaching their developmental potential. ${ }^{12}$ This inequity has profound implications for children and for LMICs. ${ }^{3}$ The WHO and UNICEF Nurturing Care Framework calls for health and social systems to support caregivers to optimise children's development potential. ${ }^{4}$ Major evidence gaps remain for caregiver support and early child development interventions led by frontline workers.

The international Guide for Monitoring Child Development (GMCD) early intervention package is a comprehensive programme based on bioecological theory for use with children 0-42 months of age. It addresses developmental difficulties using an individualised approach based on functional milestones and family strengths, rather than 
age-specific recommendations. ${ }^{5-8}$ GMCD developmental milestones for expressive and receptive language, gross and fine motor, relating, play and self-help have been validated in Argentina, India, South Africa and Turkey. ${ }^{68}$ Two expert panels have rated the GMCD as the highestperforming instrument available for monitoring and developmental support in LMICs. ${ }^{9}{ }^{10}$ The package is now available in digital format using the Android operating system.

To date, however, no clinical trials have studied the GMCD's effectiveness. In this study, we will conduct a hybrid type 1 effectiveness/implementation assessment in India and Guatemala. The study will use a nonblinded, two-arm parallel group cluster-randomised design with a planned cross-in of the control group into the intervention. The primary objectives are (a) to evaluate the effectiveness of the GMCD intervention; (b) to use an implementation science framework to understand barriers and facilitators and (c) to conduct an economic evaluation.

\section{METHODS AND ANALYSIS \\ Study setting}

We will work in rural India and Guatemala where many children under 5 years are at risk for suboptimal development $\left(84 \%\right.$ and $70 \%$, respectively). ${ }^{1}$ In Guatemala, the partner is Maya Health Alliance, a primary care organisation working with rural families of Indigenous Maya ethnicity. Each study cluster in Guatemala will consist of village-based clinical programmes coordinated by a frontline worker from Maya Health Alliance or another collaborating organisation. In India, the partner is Mahatma Gandhi Institute of Medical Sciences (MGIMS), which will work closely with Anganwadi workers in the state of Maharashtra. Anganwadi workers are frontline workers who provide services to children through the government's Integrated Child Development Services programme. Each cluster in India will consist of two Anganwadi centres and its affiliated workers.

\section{Eligibility criteria}

Families of children aged 0-24 months that are eligible to receive services from frontline workers will be eligible. Children who are critically ill and are judged by staff to require center-based care and children whose caregivers do not provide consent will be excluded.

\section{Interventions}

The GMCD early intervention package guides frontline workers through caregiver visits, using seven steps (box 1) that employ family-centred care principles, openended interviewing and mutual problem-solving skills. The GMCD intervention is available as a digital Android application, which will be used in this study.

In this trial, the GMCD intervention will be integrated into existing usual care monthly home visits delivered by frontline workers. Details of usual care will differ from
Box 1 Seven steps of the monthly Guide for Monitoring Child Development early intervention visit

Explain the importance of the early years for brain development to caregiver.

- Elicit high-priority caregiver concerns about the child's development.

- Assess the child's functioning in developmental domains using open-ended questions and standardised age-appropriate milestones.

- Explore existing psychosocial risk factors and nurturing care already provided by the family, elicit and support child and family strengths.

- Provide specific recommendations for nurturing care activities for the child's level of functioning in seven domains of development.

- Problem solve other opportunities to address child health and psychosocial risk factors.

- Set mutual goals around interventions, referrals to community resources and plans for the next visit.

cluster to cluster, but typically include health promotion activities such as growth monitoring and immunisations, and nutritional support activities such as complementary feeding education and supplementation. Individuals not enrolled in the intervention will receive all usual care activities.

Frontline workers will be trained in use of the GMCD intervention through group sessions and individual practice by certified trainers. Training will consist of 30-40 contact hours with additional time between training sessions to allow for practice of new skills. Final competence will be assessed by direct observation by GMCD trainers.

\section{Outcomes}

Based on our preliminary work, we expect to observe improvements across all developmental domains but expect the largest changes to be in language. ${ }^{11}$ Therefore, we will use change from 0 to 12 months in the language composite score of the Bayley Scales of Infant Development, 3rd edition (BSID-3) as our primary developmental outcome. ${ }^{12}$ Our secondary outcome is improvement in nurturing care. To assess this, we will measure changes from 0 to 12 months in the Home Observation for Measurement of the Environment Scale (HOME). ${ }^{13}$

\section{Participant timeline}

Participants will be assigned to usual care or the GMCD intervention for 12 months. After 12 months, the usual care arm will cross-in to the intervention, which will continue in both arms for 24 total months. A schematic of participant enrollment, study visits and assessments is given in figure 1, and key instruments are summarised in table 1. Representative data collection forms in English are given in online supplemental file 1 .

\section{Sample size and recruitment}

Our total sample size is 624 children in 52 clusters, $\mathrm{n}=312$ (26 clusters) in India and n=312 (26 clusters) in Guatemala. Our primary effectiveness outcome is to compare 


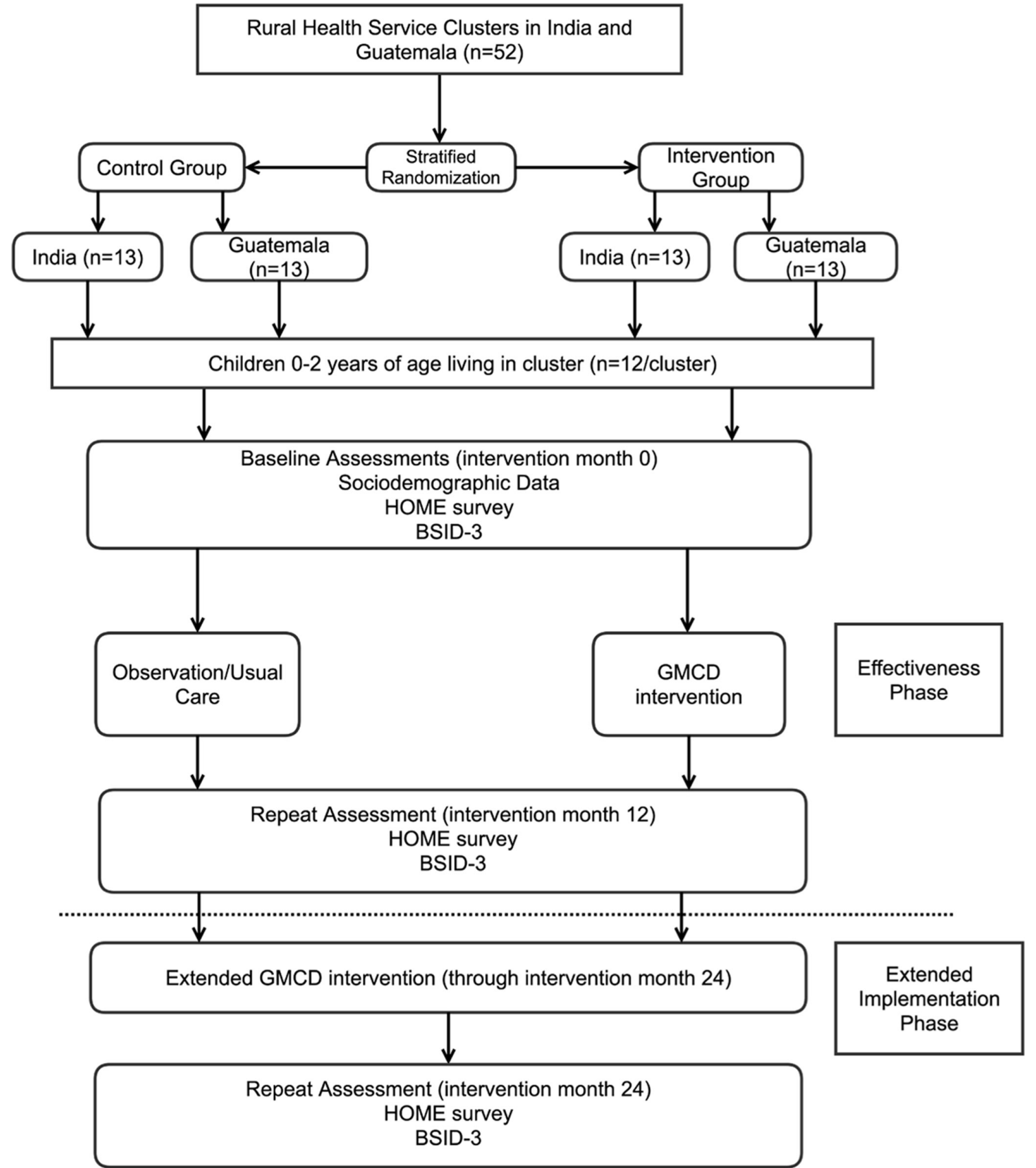

Figure 1 Schematic of study timeline and participant flow. BSID3, Bayley Scales of Infant Development, 3rd edition; GMCD, Guide for Monitoring Child Development; HOME, Home Observation for Measurement of the Environment Scale.

the difference in mean BSID3 Scores at 12 months. Cohen's d effect sizes of recent community-based integrated parenting interventions on children's development including studies in India and Guatemala have ranged from 0.3 to 0.4 SDs. ${ }^{114-17}$ Assuming an intracluster correlation coefficient of 0.2 and refusals and attrition of $25 \%$, our sample size will have $80 \%$ power to detect an overall difference of $0.3 \mathrm{SD}$ on the BSID3 language composite score. The sample size is also powered to allow independent analysis of each site at a difference of $0.4 \mathrm{SD}$ with $80 \%$ power.

Research staff will obtain lists of eligible children from participating frontline workers. Study nurses will join the frontline health worker at enrollment home visits, confirm eligibility and solicit informed consent. These recruitment activities will be supplemented with phone calls or additional home visits as needed.

\section{Allocation and blinding}

At study initiation, 52 clusters (26 each in India and Guatemala) will be randomly allocated in a 1:1 ratio to the study arms with stratification by country. Unique identifier number for clusters will be provided to the Bostonbased study statistician in lieu of names prior to randomisation, which will be constrained to provide balance on covariates of chronic child malnutrition and distance 


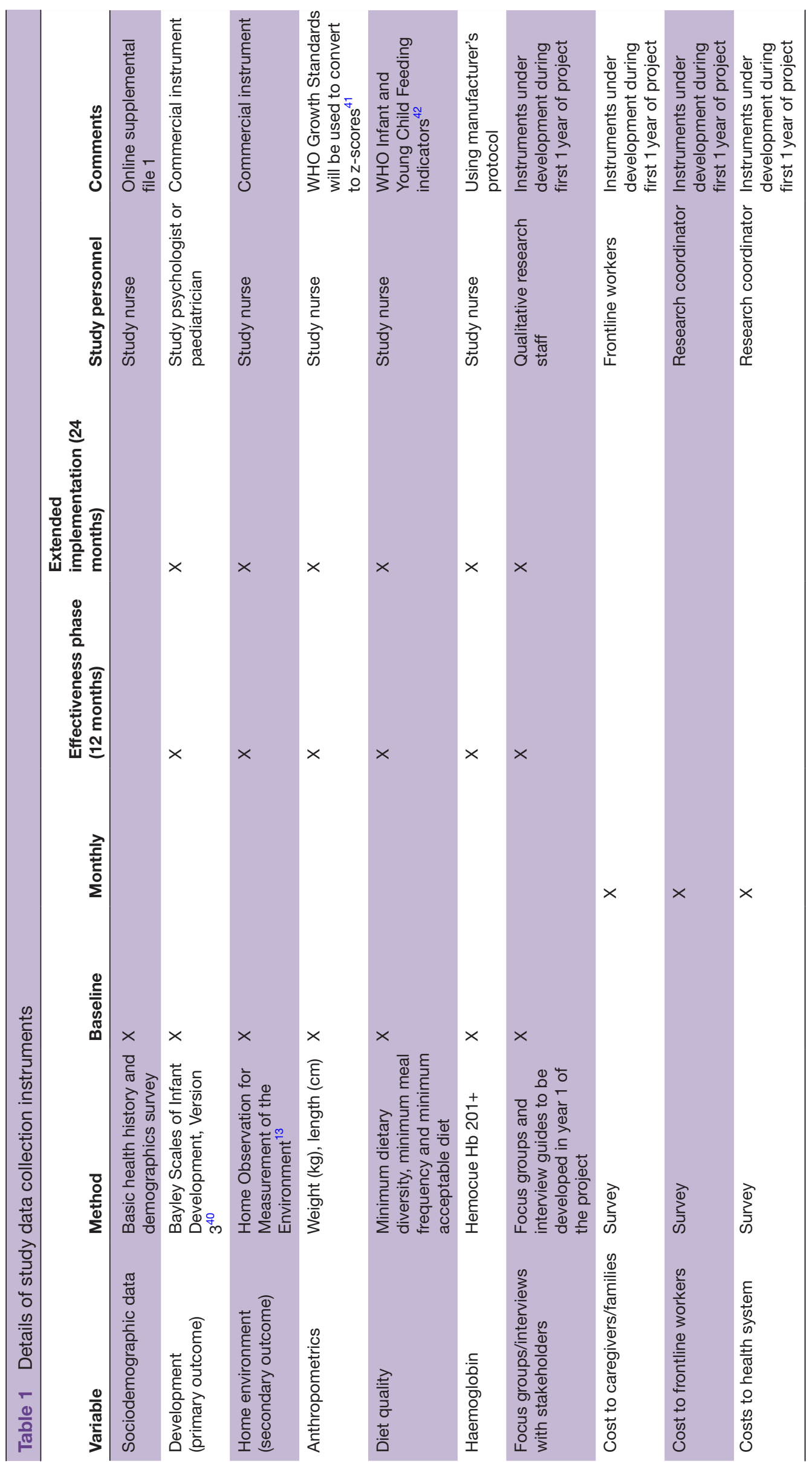


to nearest health centre. ${ }^{18}$ The statistician will generate the randomisation scheme using Stata's CVCRAND command (College Station, Texas, USA) which uses computerised randomisation sequence generation to generate and select a scheme meeting pre-determined constraint requirements.

Once the clusters have been assigned, eligible children will be randomly ordered on the list and the first six children whose caregivers consent will be enrolled for each of two age categories (0-12 months and 13-24 months, $\mathrm{n}=12$ per cluster). Only one child per family will be enrolled. All study participants in the same cluster will be assigned to the same study arm. All age eligible children within a frontline worker's coverage area will receive the intervention as caregivers wish, but data on endpoints will only be collected on study-enrolled children. Assessors conducting development tests and the study statistician will remain blinded.

\section{Data collection and management}

An outline of instruments is given in table 1. Most data will be entered online into a REDCap database (hosted by each country lead institution or Brigham and Women's Hospital).${ }^{19}$ Data entry and quality control checks on at least $10 \%$ of data will be conducted weekly by study coordinators. Study staff will collect all planned data points for agreeable participants who drop out of the intervention. The study will include haemoglobin analysis using the Hemocue Hb 201 device (www.hemocue.com) which will be acquired locally by each of the participating field sites.

\section{Primary statistical analysis}

Our main intention-to-treat analysis will assess the mean differences between arms using t-tests or Wilcoxon-MannWhitney tests (as appropriate) and regression modelling of BSID scaled composite scores using the following mixed-effects model:

$$
Y_{i j}=\beta_{0}+\delta X_{i j}+u_{i}+e_{i j}
$$

where $Y_{i j}$ is the BSID composite scaled score of participant $j$ in cluster $i$; $\delta=$ treatment effect of interest (difference between group mean BSID Scores); $X=$ cluster assignment, and $u$ and $e$ are random intercepts at cluster and participant levels, respectively. Results will be reported as the difference in scores between groups. We will conduct sensitivity analyses controlling for any remaining baseline imbalances at participant or cluster level. Because mixed effects models are robust to data missing at random (MAR), we will assess data missingness. ${ }^{20}$ If missing data is not MAR, we will conduct multilevel multiple imputation.

\section{Implementation and qualitative analyses}

We will use the Reach, Effectiveness, Adoption, Implementation, Maintenance (RE-AIM) framework to assess implementation outcomes as outlined in figure $2 .{ }^{21} \mathrm{We}$ will also conduct explanatory qualitative analyses to
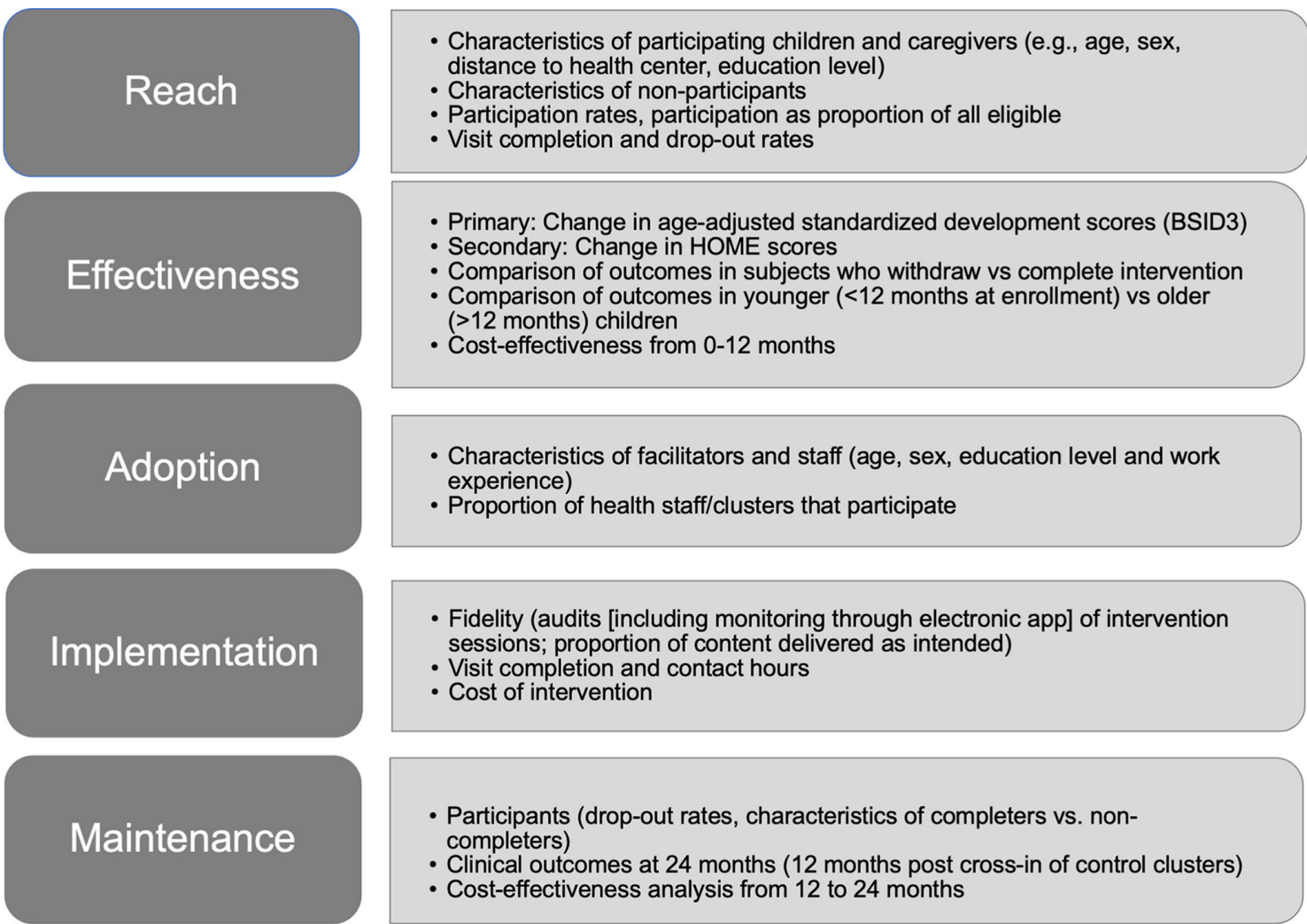

Figure 2 Description of RE-AIM dimensions and related indicators to be assessed. BSID3, Bayley Scales of Infant Development, 3rd edition; HOME, Home Observation for Measurement of the Environment Scale; RE-AIM, Reach, Effectiveness, Adoption, Implementation, Maintenance framework. 
Table 2 Consolidated Framework for Implementation Research constructs planned for use in comparative site analysis

\begin{tabular}{ll}
\hline Domain & Construct \\
\hline Intervention & Adaptability \\
characteristics & Complexity \\
& Cost \\
& Design quality \\
& Evidence strength/quality \\
& Intervention source \\
& Perceived scalability and sustainability \\
& Relative advantage \\
& Collective efficacy \\
& Culture \\
Inner setting & Implementation climate \\
& Networks and communication \\
& Structural characteristics \\
& Readiness for implementation \\
& Team characteristics \\
& Community characteristics \\
& External policies/incentives \\
Outer setting & Decision-making \\
Process & Engaging \\
\hline System & Resource continuity \\
characteristics & System architecture \\
& Strategic policy alignment \\
\hline
\end{tabular}

determine the factors that distinguish high-performing and low-performing clusters in both sites. These analyses will be guided by Consolidated Framework for Implementation Research (CFIR) constructs, principally from the Intervention Characteristics and Inner Settings domains which are especially relevant for our early-stage hybrid type 1 trial design (table 2). ${ }^{22} 23$

To conduct these analyses, $6-8$ clusters in each site will be identified based on differences on RE-AIM measures. Subsequently, we will conduct in-depth interviews and focus group discussions with implementing providers. An interview codebook will be constructed following CFIR constructs and transcripts will be double coded. After this, two coders will rate the CFIR constructs to reflect their positive or negative influence and the strength of each for distinguishing high and low performance. ${ }^{24}$

\section{Economic evaluation}

We will perform a costing exercise and cost-effectiveness analysis as previously described. ${ }^{25-29}$ System-level cost assessments will be structured around the WHO framework for health systems. ${ }^{30}$ Costs incurred by frontline personnel will include time or money spent in training and evaluation sessions. Costs incurred by caregivers and families will include time spent on GMCD visits.

Incremental costs of the interventions compared with control will be generated using multilevel regression analysis with generalised linear models (for skewed cost data and clustering effects). ${ }^{31-34}$ To provide mean and 95\% CIs for incremental costs, we will use non-parametric methods based on bootstrapped estimates of mean costs. ${ }^{35}{ }^{36}$ A discount rate of $3 \%$ will be applied to costs, adjusted between $0 \%$ and $6 \%$ for sensitivity tests. The same strategy will be used to obtain incremental effectiveness and cost-effectiveness ratios.

\section{ETHICS AND DISSEMINATION}

\section{Data monitoring and safety}

As an unblinded trial of minimal risk, the principal study investigators will review study progress and safety. Oversight by an independent Data Safety Monitoring Board is not planned. No stopping rules are planned. Principal investigators or key delegates will audit trial data, including subject accrual and status, compliance with study procedures, complaints and protocol deviations on at least a monthly basis with reports to overseeing ethics committees.

\section{Patient and public involvement}

Patients and the public were not involved in the development of the protocol. Investigators will hold dissemination meetings with community leaders in each site to discuss interim lessons learnt and final outcomes.

\section{Ethics approvals, risks, benefits}

The study has been approved by the Institutional Review Boards of Brigham and Women's Hospital (2020P002143), MGIMS (IEC/COMMED/105/2020) and Maya Health Alliance (WK 2020 003); and by the Indian Council of Medical Research/Health Ministry Screening Committee (2020-10139). Any protocol changes will be approved by the above committees.

This project involves a minimal risk intervention, and adverse intervention-related outcomes are not anticipated. During the capillary blood sample collection for haemoglobin assessment, there is risk of temporary discomfort to the child and rare risk of infection. For caregiver participants, risks include lost productivity because of time requirements and psychological distress from discussing any potential or observed developmental delays in their children. An individual experiencing adverse health outcomes while participating in the study will be referred to clinical care by the study team. Compensation or defrayment of medical costs will not be provided. Children and caregivers in the clinical trial in both control and intervention arms will benefit from access to a panel of developmental tests which they otherwise may not have access to. The intervention arm (and the control arm after 12 months) will receive intensive individualised support to promote early child development.

\section{Informed consent and confidentiality}

Consent will be at the individual caregiver level, not the cluster level. In Guatemala, study staff members will use a verbal informed consent script. There is emerging international consensus that verbal informed consent is 
appropriate in contexts where signed informed consent may not be culturally appropriate. ${ }^{37}$ In Guatemala, the primary ethics committee encourages verbal informed consent given the legacy of historical trauma and discrimination against indigenous populations and low levels of literacy. ${ }^{38}$ During the Guatemalan civil war, individuals who were not literate could be made to sign documents as an oppressive strategy for extracting resources and confessions. ${ }^{39}$ This study will not replicate those potentially traumatic practices. All consent procedures will occur in the language of the participant's choosing. Research staff in Guatemala will be natively fluent in local Mayan languages and Spanish. Study staff member will record the date of verbal consent and provide a copy of the script to the caregiver.

In India, study staff members will read the informed consent, or the caregiver will be given time to read the document, according to preference. All consent procedures will occur in the language of the participant's choosing. Research staff in India will be natively fluent in Marathi and Hindi. After reading the document, if the caregiver agrees to participate, they will sign the consent document and receive a copy.

To protect confidentiality, all paper research forms will be kept in locked file cabinets. Most data from study visits will be entered directly online, with entries linked only to subject identifying numbers. Once data extraction and cleaning has been completed, the analysis phase will be de-identified.

\section{Dissemination policy}

All research results will be made available in the public domain. The principal investigator and the co-investigators will ensure that these are disseminated through presentations at national (India, Guatemala) and international conferences. Authorship on publication will follow ICMJE guidelines.

We will share variable dictionaries, definitions and de-identified data from clinical trial participants. The lack of sensitive data elements in the dataset, the remote locations and de-identification procedures make deduction of study participants unlikely. We will deposit the data in a suitable public data repository, and it will be freely available there for any researcher who adheres to the procedures of the repository. We will submit datasets no later than 2 years after the publication of the main study paper.

\footnotetext{
Author affiliations

${ }^{1}$ Department of Community Medicine, Dr. Sushila Nayar School of Public Health, Mahatma Gandhi Institute of Medical Sciences, Sevagram, Maharashtra, India ${ }^{2}$ Department of Pediatrics, Acibadem Maslak Private Hospital, Istanbul, Turkey ${ }^{3}$ Ummeed Child Development Center, Mumbai, Maharashtra, India ${ }^{4}$ Center for Indigenous Health Research, Wuqu' Kawoq I Maya Health Alliance, Tecpán, Chimaltenango, Guatemala

${ }^{5}$ Developmental-Behavioral Pediatrics Division, Department of Pediatrics, Faculty of Medicine, Ankara University, Ankara, Turkey

${ }^{6}$ Proyecto Aigle, Guatemala, Guatemala

${ }^{7}$ Division of Global Health Equity, Brigham and Women's Hospital, Boston, Massachusetts, USA
}

${ }^{8}$ Department of Global Health and Social Medicine, Harvard Medical School, Boston, Massachusetts, USA

${ }^{9}$ Global Health and Social Medicine, Harvard Medical School, Boston,

Massachusetts, USA

${ }^{10}$ Department of Global Health, University of Washington, Seattle, Washington, USA

${ }^{11}$ Department of Epidemiology, University of Washington, Seattle, Washington, USA

Contributors AC, IE, MdPG, SG, VK, CL, ACM, BHW, PR contributed to conceptualisation. PR contributed to funding acquisition. AR, RM, RS, AC, SG, $\mathrm{CL}, \mathrm{CM}, \mathrm{ACM}, \mathrm{BHW}$, PR contributed to methodology. AR, RS, MdPG, SG, CM, PR contributed to supervision. AR, PR contributed to writing, original draft. AR, RM, RS, AC, IE, MdPG, SG, VK, CL, CM, ACM, BHW contributed to writing, review and editing.

Funding USA National Institutes of Health/Eunice Kennedy Shriver National Institute of Child Health and Human Development, 1R01HD100984-01A1.

Disclaimer The study sponsor will not be involved in study design; collection, management, analysis, and interpretation of data; writing of reports; or the decision to submit reports for publication.

Competing interests No, there are no competing interests.

Patient consent for publication Not required.

Ethics approval Brigham and Women's Hospital (2020P002143), Mahatma Gandhi Institute of Medical Sciences (IEC/COMMED/105/2020), Maya Health Alliance (WK 2020 003), Indian Council of Medical Research/Health Ministry Screening Committee (2020-10139).

Provenance and peer review Not commissioned; internally peer reviewed.

Data availability statement Data are available in a public, open access repository.

Supplemental material This content has been supplied by the author(s). It has not been vetted by BMJ Publishing Group Limited (BMJ) and may not have been peer-reviewed. Any opinions or recommendations discussed are solely those of the author(s) and are not endorsed by BMJ. BMJ disclaims all liability and responsibility arising from any reliance placed on the content. Where the content includes any translated material, BMJ does not warrant the accuracy and reliability of the translations (including but not limited to local regulations, clinical guidelines, terminology, drug names and drug dosages), and is not responsible for any error and/or omissions arising from translation and adaptation or otherwise.

Open access This is an open access article distributed in accordance with the Creative Commons Attribution Non Commercial (CC BY-NC 4.0) license, which permits others to distribute, remix, adapt, build upon this work non-commercially, and license their derivative works on different terms, provided the original work is properly cited, appropriate credit is given, any changes made indicated, and the use is non-commercial. See: http://creativecommons.org/licenses/by-nc/4.0/.

ORCID iDs

Abhishek Raut http://orcid.org/0000-0001-5671-5329

Revan Mustafayev http://orcid.org/0000-0003-1065-684X

Roopa Srinivasan http://orcid.org/0000-0001-9268-7396

Ilgi Ertem http://orcid.org/0000-0001-9187-0869

Subodh Gupta http://orcid.org/0000-0002-4412-9389

Chunling Lu http://orcid.org/0000-0002-4780-9451

Ann C Miller http://orcid.org/0000-0001-6841-9439

Peter Rohloff http://orcid.org/0000-0001-7274-8315

\section{REFERENCES}

1 Lu C, Black MM, Richter LM. Risk of poor development in young children in low-income and middle-income countries: an estimation and analysis at the global, regional, and country level. Lancet Glob Health 2016;4:e916-22.

2 McCoy DC, Peet ED, Ezzati M, et al. Early childhood developmental status in low- and middle-income countries: national, regional, and global prevalence estimates using predictive modeling. PLoS Med 2016;13:e1002034.

3 Richter LM, Daelmans B, Lombardi J, et al. Investing in the foundation of sustainable development: pathways to scale up for early childhood development. Lancet 2017;389:103-18.

4 Britto PR, Lye SJ, Proulx K, et al. Nurturing care: promoting early childhood development. Lancet 2017;389:91-102.

5 Ertem IO. The international Guide for Monitoring Child Development: enabling individualised interventions. Early Childhood Matters 2017:83-8. 
6 Ozturk Ertem I, Krishnamurthy V, Mulaudzi MC, et al. Validation of the international Guide for Monitoring Child Development demonstrates good sensitivity and specificity in four diverse countries. Acta Paediatr 2019;108:1074-86.

7 Ertem IO, Dogan DG, Gok CG, et al. A Guide for Monitoring Child Development in low- and middle-income countries. Pediatrics 2008;121:e581-9.

8 Ertem IO, Krishnamurthy V, Mulaudzi MC, et al. Similarities and differences in child development from birth to age 3 years by sex and across four countries: a cross-sectional, observational study. Lancet Glob Health 2018;6:e279-91.

9 Boggs D, Milner KM, Chandna J, et al. Rating early child development outcome measurement tools for routine health programme use. Arch Dis Child 2019;104:S22-33.

10 Fischer VJ, Morris J, Martines J. Developmental screening tools: feasibility of use at primary healthcare level in low- and middleincome settings. J Health Popul Nutr 2014;32:314-26.

11 Martinez B, Cardona S, Rodas P, et al. Developmental outcomes of an individualised complementary feeding intervention for stunted children: a substudy from a larger randomised controlled trial in Guatemala. BMJ Paediatr Open 2018;2:e000314.

12 Black JM, Hoeft F. Utilizing biopsychosocial and strengths-based approaches within the field of child health: what we know and where we can grow. New Dir Child Adolesc Dev 2015;2015:13-20.

13 Caldwell BM, Bradley RH. Home Observation for Measurement of the Environment: administration manual. Tempe, AZ: Arizona State University, 2003

14 Luo R, Emmers D, Warrinnier N, et al. Using community health workers to deliver a scalable integrated parenting program in rural China: a cluster-randomized controlled trial. Soc Sci Med 2019;239:112545.

15 Yousafzai AK, Rasheed MA, Rizvi A, et al. Effect of integrated responsive stimulation and nutrition interventions in the Lady Health Worker programme in Pakistan on child development, growth, and health outcomes: a cluster-randomised factorial effectiveness trial. Lancet 2014;384:1282-93.

16 Vazir S, Engle P, Balakrishna N, et al. Cluster-randomized trial on complementary and responsive feeding education to caregivers found improved dietary intake, growth and development among rural Indian toddlers. Matern Child Nutr 2013:9:99-117.

17 Grantham-McGregor SM, Fernald LCH, Kagawa RMC, et al. Effects of integrated child development and nutrition interventions on child development and nutritional status. Ann N Y Acad Sci 2014;1308:11-32.

18 Moulton LH. Covariate-based constrained randomization of grouprandomized trials. Clin Trials 2004;1:297-305.

19 Harris PA, Taylor R, Thielke R, et al. Research Electronic Data Capture (REDCap)--a metadata-driven methodology and workflow process for providing translational research informatics support. $J$ Biomed Inform 2009;42:377-81.

20 Robins JM, Rotnitzky A, Zhao LP. Analysis of semiparametric regression models for repeated outcomes in the presence of missing data. J Am Stat Assoc 1995;90:106-21.

21 Holtrop JS, Estabrooks PA, Gaglio B, et al. Understanding and applying the RE-AIM framework: clarifications and resources. J Clin Trans/ Sci 2021;5:1-31.

22 Damschroder LJ, Aron DC, Keith RE, et al. Fostering implementation of health services research findings into practice: a consolidated framework for advancing implementation science. Implement Sci 2009;4:50.

23 Means AR, Kemp CG, Gwayi-Chore M-C, et al. Evaluating and optimizing the Consolidated Framework for Implementation Research (CFIR) for use in low- and middle-income countries: a systematic review. Implement Sci 2020;15:17.

24 Damschroder LJ, Lowery JC. Evaluation of a large-scale weight management program using the Consolidated Framework for Implementation Research (CFIR). Implement Sci 2013;8:51.

25 Tsai S, Ruhumuriza J, Umugiraneza G. Training manual on tracking financial data in health facilities in Kayonza and Kirehe. Boston, MA: Harvard Medical School, 2011.

26 Lu C, Tsai S, Ruhumuriza J. General guidelines on obtaining financial data for local health facilities in two districts of Rwanda. Boston, MA: Harvard Medical School, 2013.

27 Lu C, Tsai S, Ruhumuriza J, et al. Tracking rural health facility financial data in resource-limited settings: a case study from Rwanda. PLoS Med 2014;11:e1001763.

28 Lu C, Liu K, Li L, et al. Sensitivity of measuring the progress in financial risk protection to survey design and its socioeconomic and demographic determinants: a case study in Rwanda. Soc Sci Med 2017;178:11-18.

29 Lu C, Chin B, Lewandowski JL, et al. Towards universal health coverage: an evaluation of Rwanda Mutuelles in its first eight years. PLoS One 2012;7:e39282.

30 World Health Organization. Health systems: improving performance. Geneva, Switzerland: World Health Organization, 2000.

31 Goldstein H. Multilevel statistical models. 3rd edn. London: Arnold, 2003.

32 Manning WG. The logged dependent variable, heteroscedasticity, and the retransformation problem. J Health Econ 1998;17:283-95.

33 Manning WG, Mullahy J. Estimating log models: to transform or not to transform? J Health Econ 2001;20:461-94.

34 Buntin MB, Zaslavsky AM. Too much ado about two-part models and transformation?: Comparing methods of modeling Medicare expenditures. J Health Econ 2004;23:525-42.

35 Moulton LH, Zeger SL. Analyzing repeated measures on generalized linear models via the bootstrap. Biometrics 1989;45:381-94.

36 Efron B, Tibshirani R. An introduction to the bootstrap. London: Chapman and Hall, 1993.

37 Goverment US. Protection of Human Subjects: Documentation of Informed Consent 46.117.c(1)iii. Available: https://ecfr.federalregister. gov [Accessed 11 Aug 2021].

38 Cerón A, Ruano AL, Sánchez S, et al. Abuse and discrimination towards Indigenous people in public health care facilities: experiences from rural Guatemala. Int J Equity Health 2016;15:77.

39 Chary A, Rohloff P, eds. Privatization and the new medical pluralism: shifting healthcare landscapes in Maya Guatemala. Lanham: Lexington Books, 2015.

40 Bayley N. Bayley Scales of Infant and Toddler Development. 3rd edn. San Antonio, TX: Psychological Corp, 2006.

41 WHO Multicentre Growth Reference Study Group. WHO child growth standards based on length/height, weight and age. Acta Paediatr Suppl 2006;450:76-85.

42 World Health Organization. Indicators for assessing infant and young child feeding practices: definitions and measurement methods. Geneva: World Health Organization, 2021. 